\title{
Spatio-temporal Analysis of Brain MRI Images Using Hidden Markov Models
}

\author{
Ying Wang ${ }^{1}$, Susan M. Resnick ${ }^{2}$, and Christos Davatzikos ${ }^{1}$ \\ 1 Section of Biomedical Image Analysis, Department of Radiology, \\ University of Pennsylvania, Philadelphia, USA \\ ${ }^{2}$ Laboratory of Personality and Cognition, National Institute on Aging, \\ Baltimore, USA
}

\begin{abstract}
A rapidly increasing number of medical imaging studies is longitudinal, i.e. involves series of repeated examinations of the same individuals. This paper presents a methodology for analysis of such 4D images, with brain aging as the primary application. An adaptive regional clustering method is first adopted to construct a spatial pattern, in which a measure of correlation between morphological measurements and a continuous patient's variable (age in our case) is used to group brain voxels into regions; Secondly, a dynamic probabilistic Hidden Markov Model (HMM) is created to statistically analyze the relationship between spatial brain patterns and hidden states; Thirdly, parametric HMM models under a bagging framework are used to capture the changes occurring with time by decoding the hidden states longitudinally. We apply this method to datasets from elderly individuals, and test the effectiveness of this spatio-temporal model in analyzing the temporal dynamics of spatial aging patterns on an individual basis. Experimental results show this method could facilitate the early detection of pathological brain change.
\end{abstract}

\section{Introduction}

A number of advances in medical imaging technologies allow researchers to study the progression of anatomical or functional changes in a number of diseases and therapies. High-dimensionality pattern analysis methods have been increasingly used to measure imaging patterns and use them for individual diagnosis and progression. In the literature of aging and Alzheimer's Disease (AD), highdimensional classification work aims to provide diagnostic predictors for early marker of $\mathrm{AD} 1 / 234$. However, there is an increasing need for methods that aim to measure subtle gradual progression of change, especially in neurodegenerative diseases. Compared with the dichotomous classification approaches, pattern regression methods offer an alternative approach, which tries to estimate continuous variables from imaging data [5/6 7/8].

Although high-dimensionality pattern analysis methods have potential to provide biomarkers for early detection of Alzheimer's disease (AD), they typically take into account one scan at a time, which can render them less sensitive to subtle longitudinal changes that relate to disease progression. Longitudinal studies

T. Jiang et al. (Eds.): MICCAI 2010, Part II, LNCS 6362, pp. 160-168, 2010.

(C) Springer-Verlag Berlin Heidelberg 2010 
allow us to measure subtle changes more accurate by repeatedly evaluating the same subject over time 9 1011]. For example, Driscoll et al. evaluated 138 nondemented samples with longitudinal scans up to 10 years, and observed that brain volume declined at specific regions for healthy people while accelerated changes were shown in MCI group [11.

To statistically analyze temporal dynamics and capture disease progression, a general solution is spatio-temporal modeling, which has been quite widely used in pattern recognition and computer vision areas, such as speech processing and activity analysis [1213. Spatio-temporal analysis has also found applicability in measuring the temporal evolution of brain activation under fMRI studies 141516 . However, spatio-temporal analysis of brain change in longitudinal studies using advanced statistical analysis tools has been relatively scarce. Herein, we propose such an approach based on a popular dynamic model, Hidden Markov Models (HMM). First, we adaptively extract regional features by clustering brain voxels with similar correlation measurements to age. Compared with voxel-wise methods, regional measures can provide more robust and discriminative patterns. Then HMM is constructed to model the temporal evolution of brain change as a sequence of probabilistic transitions from one discrete state to the other. To improve the stability of the methodology, a bagging strategy is adopted to build ensemble HMM models and estimate state path for each subject statistically. Experiments with brain MRI serial scans from older individuals show that the regional feature-based HMM is an effective method to analyze the spatio-temporal change of brain structure. It also can potentially detect abnormal changes due to neurodegeneration, which is accomplished by comparing the individual state trajectory with changes observed in healthy elderly.

\section{Material and Methodology}

\section{$2.1 \quad$ Materials}

9-year longitudinal data with $\mathrm{T}_{1}$-weighted MRIs from 144 cognitively normal individuals $(\mathrm{CN})$ were used under BLSA study. More image acquisition details about BLSA data are described in 10 . Here, the slope of the California Verbal Learning Test (CVLT) scores for each subject over all years was used to select training samples, because CVLT test has been widely adopted for cognitive performance evaluation. These slopes were calculated by mixed-effects regression, and 58 subjects with the higher and lower CVLT score slopes were chosen for training, while the remaining 86 subjects were used for testing. The characteristics of participants in this study are shown in Table 1. In this work, brain MR scans were pre-processed to three tissue density maps, namely gray matter (GM), white matter (WM) and cerebrospinal fluid (CSF) by extensively validated techniques 28]. These three tissue density maps give a quantitative representation of the spatial tissue distribution in a template space. Brain change is reflected by volume values in the respective tissue density maps. 
Table 1. Characteristics of participants in the current study

\begin{tabular}{|c|c|c}
\hline & Training Set & Testing Set \\
\hline No. of subjects & 58 & 86 \\
\hline Gender: No. of males/females & $29 / 29$ & $49 / 37$ \\
\hline Baseline age (years) & $69.63 \pm 8.19$ & $71.04 \pm 6.82$ \\
\hline Age at last visit (years) & $76.27 \pm 8.31$ & $76.96 \pm 7.85$ \\
\hline No. of scans & & \\
Year 1 & 58 & 86 \\
Year 2 & 58 & 86 \\
Year 3 & 58 & 76 \\
Year 4 & 56 & 72 \\
Year 5 & 49 & 68 \\
Year 6 & 42 & 65 \\
Year 7 & 36 & 59 \\
Year 8 & 20 & 53 \\
Year 9 & 10 & 39 \\
Total scans & 387 & 620 \\
\hline
\end{tabular}

\subsection{Methodology}

Regional feature extraction: To generate robust patterns against measurement noise or image pre-processing errors, it is a common approach to group tissue voxels with similar characteristics. As age is the major risk factor for brain change, Pearson correlational analysis of morphological measurements and the corresponding age is adopted to measure the similarity of tissue voxels. However, given the limited number of samples, how to generate the most informative features with good generalizability is still very challenging. Leave- $k$-out bagging strategy has been proved effective in improving the robustness of measures. Given a training set $D$ of $N$ samples with longitudinal scans $k_{n}, n=1, \ldots N$, bagging procedure generates $N$ Pearson correlation coefficients between tissue values and age, by respectively sampling $D-k_{n}$ examples from the whole training set with the corresponding replacement. Then correlation confidence, defined as the quotient of the mean and variance of these correlation coefficients from the same location $u$ of tissue map $i(i=1,2,3$ represents GM, WM and CSF respectively), is used to evaluate the discrimination ability and robustness of features. The larger the absolute value of this correlation confidence is, the more relevant to brain change this feature is. Here is the mathematical formulation as follows:

where

$$
c^{i}(u)=\frac{\overline{c_{n}^{i}(u)}}{\operatorname{Var}\left(c_{n}^{i}(u)\right)}
$$

$$
c_{n}^{i}(u)=\frac{\sum_{n}\left(f_{n}^{i}(u)-\overline{f^{i}(u)}\right)\left(y_{n}-\bar{y}\right)}{\sqrt{\sum_{n}\left(f_{n}^{i}(u)-\overline{f^{i}(u)}\right)^{2} \sum_{n}\left(y_{n}-\bar{y}\right)^{2}}}
$$


$c_{n}^{i}(u)$ is the Pearson correlation coefficient between the tissue values $f_{n}^{i}(u)$ and variables $y_{n}^{i}$ (age in this work) at location $u$ of tissue map $i$ from the $n$th leave$k$-out case, from which $k$ images of the $n t h$ sample are excluded. Here, $\overline{f_{i}(u)}$ is the mean of $f_{n}^{i}(u)$ over all samples, and $\bar{y}$ is the mean of all sample ages $y_{n}$. By extensively examining voxels and their respective correlation coefficients, correlation confidence takes into account not only the discriminative ability, but also consistence of feature. The reason is that, outliers can be found via high variance of correlation coefficients, even some correlation coefficients are high at the location $u$ from cross-validation procedure.

We adopted the method of [8] and partitioned the brain into clusters of relatively homogeneous correlation with age. Given these regional clusters, features can be extracted by some statistical analysis on the respective brain regions. In order to produce a small size of effective features for efficient parameter optimization of spatio-temporal model, a feature subset was selected by the ranking criterion, which was the absolute value of leave- $k$-out correlation confidence defined as formula (1).

Spatio-temporal model: To model the brain structure change over time, it is reasonable to employ a dynamic model with probability distributions, which indicates the spatio-temporal relationship between the observed brain pattern sequences and hidden state trajectories. Since brain change in old adults usually accompanies tissue volume decline irreversibly, Markov process, in which past observations explicitly influence present measurements, is an appropriate representation for the true brain change. Therefore, HMM model with a Markov process combing unobserved state is applied to statistically analyze the spatial brain changes in longitudinal progression, then explain them by the corresponding state at each time point individually.

Mathematically, an HMM is defined by a finite set of $J$ states, and transitions among the states are governed by a set of probabilities called transition probabilities, $\alpha_{i, j}=P\left(S_{t}=j \mid S_{t-1}=i\right), 1 \leq i, j, \leq J$. Considering the gradual progression of brain structure with age, a continuous probability density function is employed to characterise the relationship between states and brain change measured with regional patterns. Specifically, a weighted sum of $M$ Gaussian distributions is commonly adopted to approximate the probability density function $b_{j}=\sum_{m=1}^{M} c_{j m} N\left(\mu_{j m}, \Sigma_{j m}, o_{t}\right)$, in which $c_{j m}$ represent weighting coefficients with stochastic constrains $\sum_{m}^{M} c_{j m}=1,1 \leq j \leq J, \mu$ and $\Sigma$ are the mean vectors and covariance matrices. $\pi(j)=P\left(S_{1}=j\right)$ is the initial probability of hidden states.

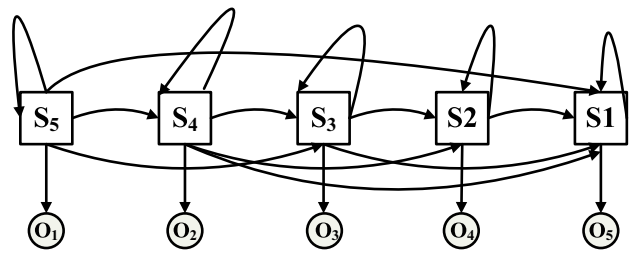

Fig. 1. The left-to-right HMM with 5 states 
Our approach aims to characterize progression along a direction, e.g. aging, disease progression or treatment effects. We therefore explicitly incorporate an additional constraint in the model, and introduce a left-to-right HMM structure with 5-states. The state index of left-to-right HMMs decreases or remains the same as time increases, as illustrated in Figure 1. To simplify the model, observation density function $b_{j}$ is represented by a single Gaussian distribution for each state $j$ in this paper.

A bagging strategy is also used to improve the model generalizability. For each leave- $k$-out loop, we build a HMM model with the associated parameters $\left(\pi_{n}, \alpha_{n}, b_{n}\right)$. Given the estimated model parameters, Viterbi decoding is employed to find the most likely state path for both training and testing sequences. In order to interpret the state transition, once $N$ state paths are obtained under bagging procedure for each subject, the state that occurs most frequently at each time point is chosen as the final state mode. A framework of the spatio-temporal modelling and analysis is shown in Figure 2 .

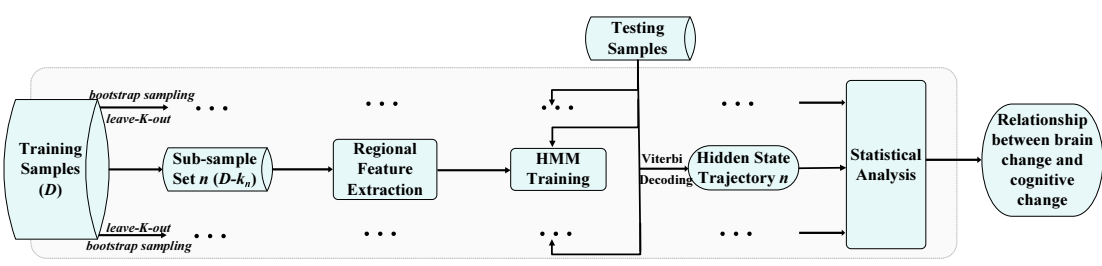

Fig. 2. A bagging framework of HMM modeling and result analysis

\section{Results and Discussion}

Given the bagging procedure, we summarized the average age distributions of the respective state index values, which were decoded by Viterbi algorithm based on all ensemble HMM models. Overall, the estimated state index value closely correlates with age in both training and testing sets as shown in Figure 3, where the state values decrease with increasing age.

To further evaluate the performance of HMM models, the state transition path for each individual was constructed. We note that almost half of them experienced the state transitions with advancing age, and several representative CNs are illustrated in Figure 4 .

In order to investigate the relationship between state transition and cognitive performance decline, CN individuals in the group with positive CVLT score slopes (referred to as Non-Cognitive Decline group, i.e. NCD) were compared with the remaining samples with negative slopes (referred to as Cognitive Decline group, i.e. CD). We examined the number of state transitions for each subject from the training set and the whole set, as illustrated in the first two plots of Figure 5. Though nearly half of them had brain structure change as expected, a much larger proportion of the $\mathrm{CN}$ individuals remained structurally stable 

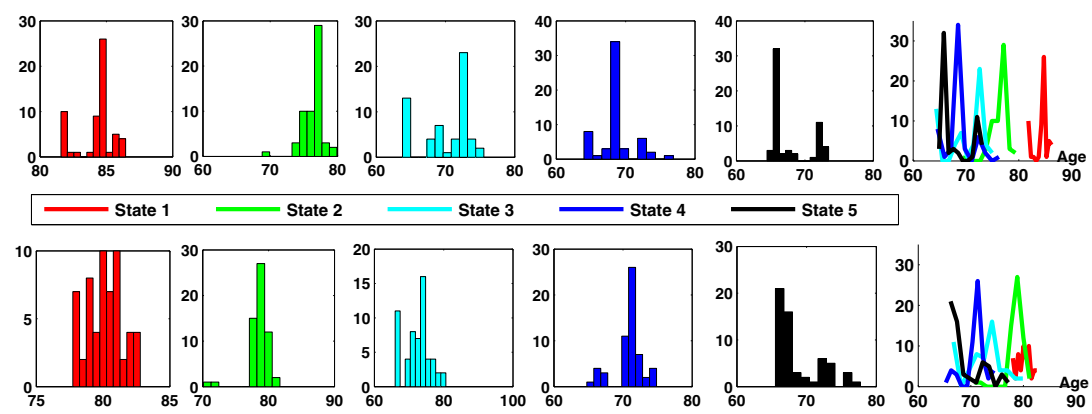

Fig. 3. Average age distributions against the respective hidden states from ensemble HMM models. The top subplots show the average age distributions of training set while the bottom subplots are the results for testing set. The final plots for each set illustrate the combination of individual average age distributions of five states.
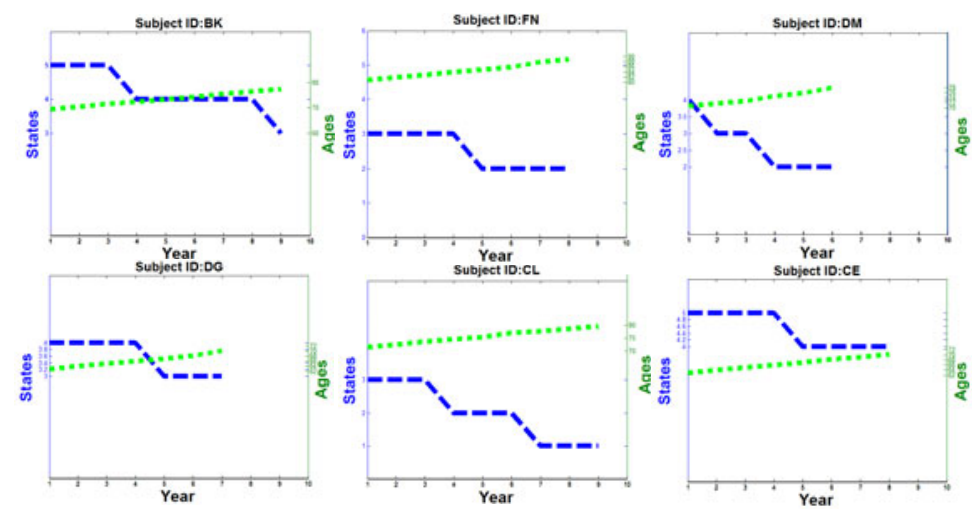

Fig. 4. Individual state paths of several samples in experiments

without any state transition. We also can see that the number of stable subjects in NCD group was larger than that of CD group. To determine which state the stable individuals remained in, we plotted the histogram of the number of state index values for those individuals without transition (the last graph in Figure 5). Notably, what was common in most "CD" subjects was that they showed relatively higher prevalence of states 1-2, which are states with the most abnormal structure, even though they remained relatively stable throughout the follow-up period.

To further understand the difference of the age-related brain changes between "relatively healthy" set and "progressive" set, we examined the average ages for the corresponding states from those two groups, respectively. Here, the samples with relatively minimal CVLT slopes and also high CVLT scores at baseline scan ( score > 45) were included into the "relatively healthy" set, while those with rapidly decreasing CVLT slopes were the "progressive" set (slope $<-0.5$ ). From 

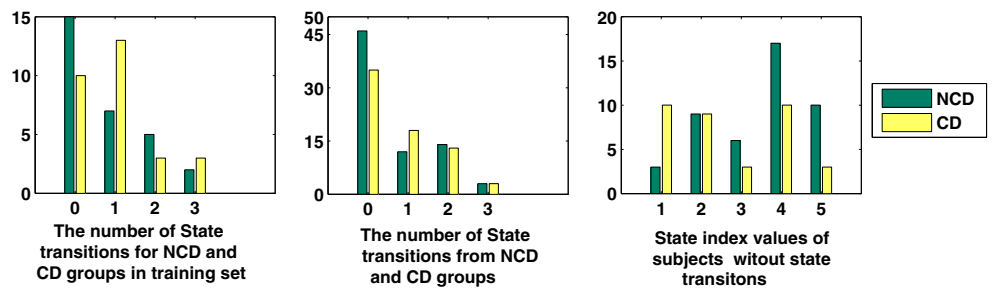

Fig. 5. Statistics of the number of state transitions for NCD and CD groups

Table 2, at states 1 and 2 (which are the most abnormal states), "progressive" group members were younger than those from "relatively healthy" group. It is possible that the "progressive" subjects in these states are the ones with most aggressively evolving disease. In addition, the age difference between the "normal" state 5 and the "abnormal" state 1 in "progressive" group was less than that of the healthy group. For "progressive" group, there was no significant difference of the mean ages between state 2 and 3. This implied that "progressive" individuals progress nonlinearly.

Table 2. Average ages against states

\begin{tabular}{|c|c|c|c|c|c|}
\hline Average Age State & State 1 & State 2 & State 3 & State 4 & State 5 \\
\hline Relatively healthy group & $\mathbf{8 6 . 7 3}$ & $\mathbf{7 7 . 8 1}$ & 70.54 & 67.11 & 65.96 \\
\hline Progressive group & 83.34 & 73.59 & 71.67 & 71.16 & 69.44 \\
\hline
\end{tabular}

Indeed, we found that several individuals demonstrated lower index values of state $1 / 2$ throughout observing period, even though they had high cognitive test scores at baseline, and were relatively younger compared with the average ages of these two states $(1 / 2)$ as illustrated in Figure 3 . Figure 6 shows the state paths with increasing age of two detected samples.
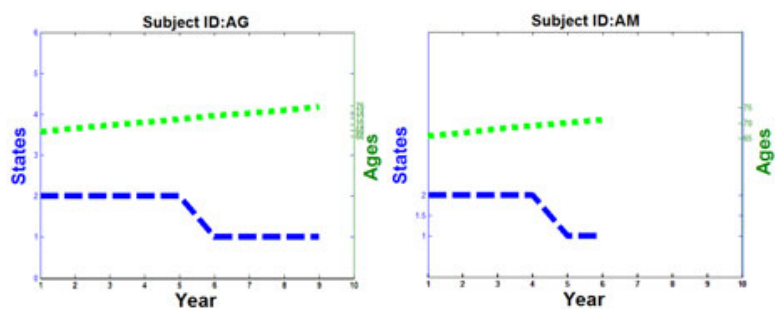

Fig. 6. State paths of two samples with abnormal decline structurally

Therefore, the individuals with early "abnormal" state paths might develop cognitive impairment in the near future. To some extent, this is consistent with 
most of MRI studies, i.e., there is an accelerating rate of change among AD-like people, even for those who seem to be cognitively healthy until later disease stages. One of the potentials of HMM models is that they might help identify early state transitions in individuals bound to later develop a disease, and therefore to intervene early enough in the process.

The distinctive patterns of brain change in aging used in HMM modeling are shown in Figure 7. We can see that most of these regional patterns are primarily located at hippocampus, superior temporal gyrus, frontal lobe, cingulate region and precuneus, which are largely in agreement with previous findings [10]1].

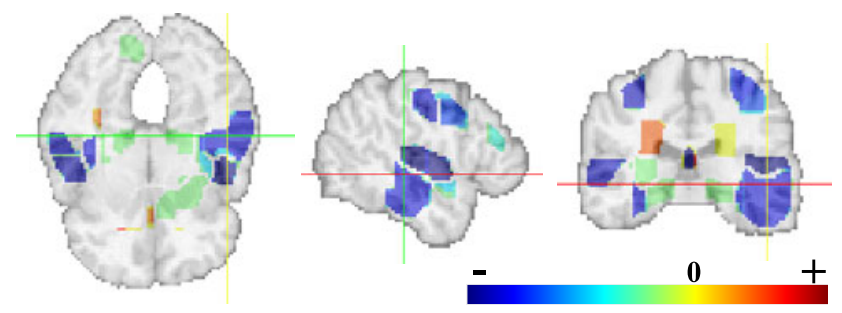

Fig. 7. Regional patterns with brain aging over time are shown by the color-coded ranking score, correlation confidence

\section{Conclusion}

We presented a methodology for analysis of longitudinal image data, and applied it to serial MRI brain scans of an aging cohort. In view of the statistical analysis between cognitive performance and brain change, we found that considerably more subjects with state transitions came from CD group. Moreover, subjects without state transitions from CD group showed high prevalence of state 1 or 2, which implied their cognitive performance might have already declined before the first visit. In addition, subjects with quickly declining cognitive performance showed faster and nonlinear brain change, compared with "relatively healthy" subjects. These findings are largely consistent with previous studies. Therefore, the HMM model is a promising approach to evaluate the spatio-temporal progression of brain change over time individually. In this work, model parameters were learned automatically from the data. However, the HMM structure was defined manually. This was mainly due to the small size of the data set, which will be addressed in future with the availability of larger data set.

\section{References}

1. Golland, P., et al.: Discriminative analysis for image-based studies. In: Dohi, T., Kikinis, R. (eds.) MICCAI 2002. LNCS, vol. 2488, pp. 508-515. Springer, Heidelberg (2002)

2. Fan, Y., et al.: Compare: Classification of morphological patterns using adaptive regional elements. IEEE Transactions on Medical Imaging 26, 93-105 (2007) 
3. Kloppel, S., et al.: Automatic classification of MRI scans in Alzheimer's disease. Brain 131(3), 681-689 (2008)

4. Vemuri, P., et al.: MRI and CSF biomarkers in normal, MCI, and AD subjects: Diagnostic discrimination and cognitive correlations. Neurology 73, 287-293 (2009)

5. Duchesne, S., et al.: Relating one-year cognitive change in mild cognitive impairment to baseline MRI features. NeuroImage 47(4), 1363-1370 (2009)

6. Ashburner, J.: A fast diffeomorphic image registration algorithm. NeuroImage 38(1), 95-113 (2007)

7. Franke, K., Ziegler, G., Klöppel, S., Gaser, C.: Estimating the age of healthy subjects from t1-weighted mri scans using kernel methods: Exploring the influence of various parameters. Neuroimage 50(3), 883-892 (2010)

8. Wang, Y., et al.: High-dimensional pattern regression using machine learning: From medical images to continuous clinical variables. NeuroImage 50(4), 1519 $1535(2010)$

9. Sullivan, E.V., et al.: Differential rates of regional brain change in callosal and ventricular size: a 4-year longitudinal mri study of elderly men. Cereb. Cortex 12, 438-445 (2002)

10. Resnick, S.M., et al.: Longitudinal magnetic resonance imaging studies of older adults: A shrinking brain. The Journal of Neuroscience 23, 3295-3301 (2003)

11. Driscoll, I., et al.: Longitudinal pattern of regional brain volume change differentiates normal aging from MCI. Neurology 72(22), 1906-1913 (2009)

12. Rabiner, L.R.: A tutorial on hidden markov models and selected applications in speech recognition. In: Proceedings of the IEEE, pp. 257-286 (1989)

13. Moeslund, T.B., et al.: A survey of advances in vision-based human motion capture and analysis. Comput. Vis. Image Underst. 104(2), 90-126 (2006)

14. Katanoda, K., Matsuda, Y., Sugishita, M.: A spatio-temporal regression model for the analysis of functional mri data. NeuroImage 17(3), 1415-1428 (2002)

15. Woolrich, M., et al.: Fully bayesian spatio-temporal modeling of fmri data. IEEE Transactions on Medical Imaging 23(2), 213-231 (2004)

16. Quirós, A., Diez, R.M., Gamerman, D.: Bayesian spatiotemporal model of fmri data. NeuroImage 49(1), 442-456 (2010) 